\title{
Treatment of Acute Appendicitis in the Patients With Severe Neutropenia
}

\author{
Hyun-Jong Kang ${ }^{\mathrm{a}}$, Sang-Yoon Kang ${ }^{\mathrm{a}}$, Won-Kyung Kang ${ }^{\mathrm{a}, \mathrm{c}}$, \\ Dong-Gun Lee ${ }^{\mathrm{b}}$, Jong-Wook Lee ${ }^{\mathrm{b}}$
}

\begin{abstract}
When appendicitis occurs in neutropenic adult patients, clinicians must balance the timing and the risks of surgery. Urgent surgery may lead to high operative complications and mortality. This case report details the treatment of appendicitis in neutropenic patients with hematologic disease. Patient 1 was a 31 years old female with severe aplastic anemia and patient 2 was a 34 years old male with AML M6. Both patients' abdominal CT scan was suggestive of appendicitis. We prepared supportive care first, delayed surgery was successfully completed without acute or late complications. We suggest that, in patients in a neutropenic state, delayed elective operation is superior to urgent operation, except in the case of lifethreatening complications such as peritonitis.
\end{abstract}

Keywords: Acute appendicitis; Severe neutropenia; Hematologic disease

\section{Introduction}

When appendicitis occurs, even if it is not at the most common site of infection in neutropenic adult patients, clinicians must balance the timing and the risks of surgery. Due to the surgical approach depends on early diagnosis and the patient's immune system, the mortality and morbidity of surgical intervention is high $[1,2]$. In general, the classic symptom of appendicitis is migrating RLQ localized abdominal pain, anorexia, nausea, vomiting, and so on. In neutropenic

\footnotetext{
Manuscript accepted for publication April 26, 2013

${ }^{\mathrm{a}}$ Department of Surgery, College of Medicine, The Catholic University of Korea, Korea

${ }^{b}$ Department of Internal Medicine, College of Medicine, The Catholic University of Korea, Korea

${ }^{\mathrm{c}}$ Corresponding author: Won-Kyung Kang, Department of Surgery, Seoul St. Mary's Hospital, Catholic University of Korea, 222, Banpodaero, Seocho-gu, Seoul 137-701, Republic of Korea.

Email: wonkkang@catholic.ac.kr
}

doi: http://dx.doi.org/10.4021/jmc1286w patients, classical symptoms may not be present since the body may not be able to mount an adequate inflammatory response. Thus, a diagnosis of acute appendicitis may be delayed or even missed [3-5]. Moreover, urgent surgery may lead to high operative complications and mortality. This case report details the diagnosis and treatment of appendicitis in neutropenic patients with hematologic disease.

\section{Case Report}

\section{Case 1}

Patient 1 was a 31-year-old Korean female who presented with a fever and sore throat in April 2012. Laboratory tests showed pancytopenia with a white blood cell count of 1.2 $\times 10^{9} / \mathrm{L}$, hemoglobin of $7.9 \times 10^{9} / \mathrm{L}$, a platelet count of 17 $\times 10^{9} / \mathrm{L}$, and an absolute neutrophil count (ANC) of 0.04 $\times 10^{9} / \mathrm{L}$. Results of a bone marrow aspiration and biopsy were compatible with severe aplastic anemia, and the patient was scheduled to receive a bone marrow transplantation. She developed diffuse abdominal pain, and an abdominal examination revealed direct tenderness, especially at McBurney's point. An urgent computed tomography (CT) scan was performed. The thickened appendix wall and cecal base was suggestive of appendicitis. However, because of her severe neutropenic state, broad-spectrum empirical antibiotic therapy following IV fluid therapy was given. Over the course of antibiotic therapy, the intensity of the patient's pain decreased and her general condition improved. Subjects were recruited for a blood component transfusion. In the course of treatment for aplastic anemia, 13 days after the first attack, the patient developed diffuse abdominal pain and diarrhea. A CT scan showed a periappendiceal abscess with increasing periappendiceal fat infiltration. After adequate preoperative preparation, particularly the blood component transfusion, the patient underwent an elective laparoscopic appendectomy (Fig. 1). The patient was able to take oral fluids on postoperative day (POD) 4. No complications occurred. The patient was discharged on POD 6. After discharge, she underwent an allogeneic bone marrow transplant and continued her scheduled therapy. 

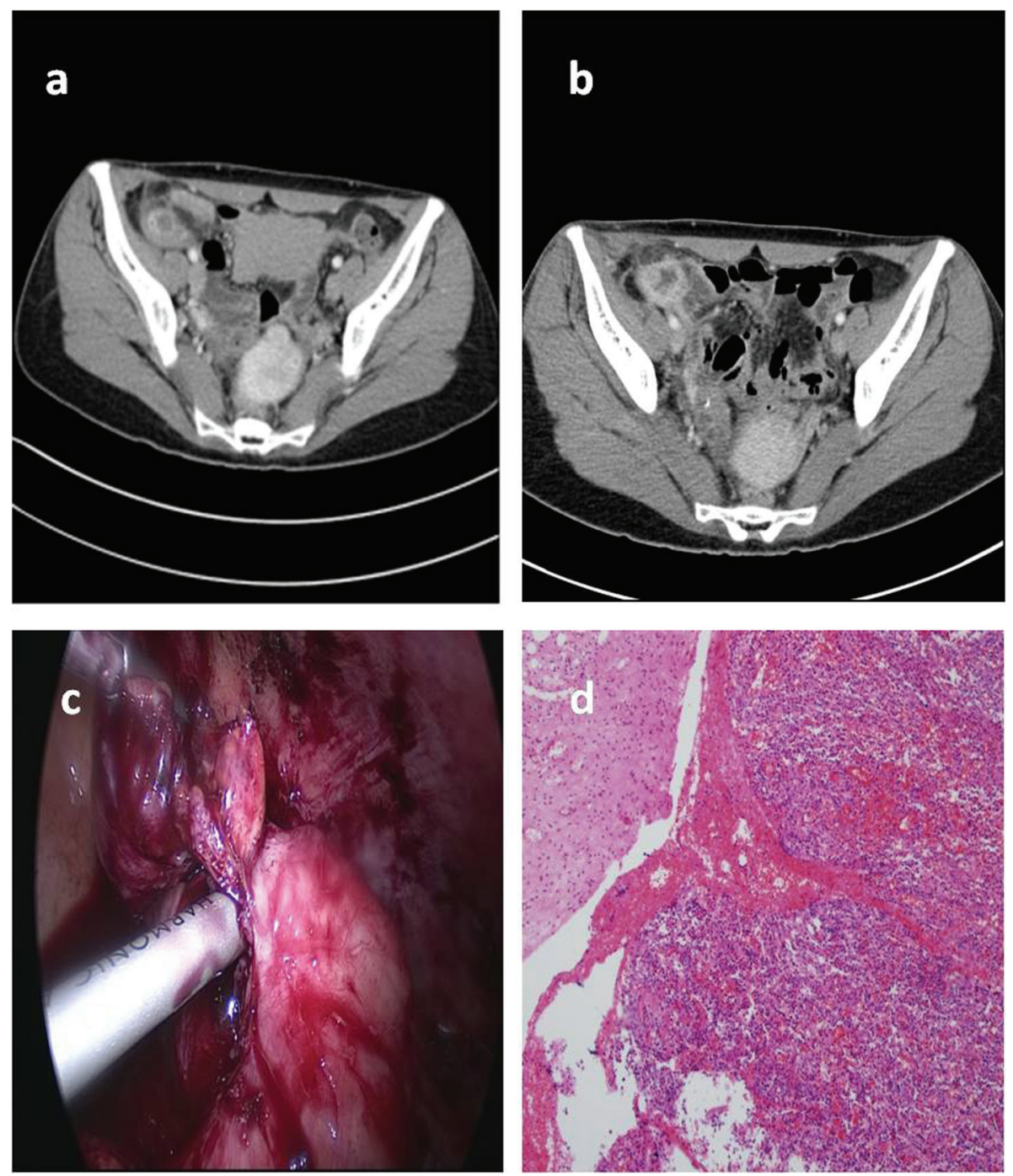

Figure 1. Radiologic, laparoscopic and histologic features of acute appendicitis in an neutropenic patient. Abdominal CT showed wall thickening and edema of the appendix and cecum (a). Abdominal CT showed periappendiceal abscess with increasing periappendiceal and pericecal fat infiltration after relapsed abdominal pain (b). A laparoscopic appendectomy was performed (c), and histologic examination of the appendix revealed infiltration of fibrin and inflammatory cells (hematoxylin-eosin, low magnification $\times 100)(d)$.

\section{Case 2}

Patient 2 was a 34-year-old Korean male who presented in July 2012 with a cold that would not improve. His complete blood count $(\mathrm{CBC})$ showed pancytopenia with a white blood cell of $2.78 \times 10^{9} / \mathrm{L}$, hemoglobin of $8.8 \times 10^{9} / \mathrm{L}$, a platelet count of $50 \times 10^{9} / \mathrm{L}$, and an ANC of $1.78 \times 10^{9} / \mathrm{L}$. The WBC differential was significant for $30 \%$ of blasts. The results of a bone marrow aspiration and biopsy were compatible with AML-M6. The results of cytogenetic analysis of the bone marrow sample demonstrated $t(6 ; 9)(\mathrm{p} 23 ; \mathrm{q} 34)$. The patient received induction chemotherapy with cytarabine. During remission induction chemotherapy, the patient experienced abdominal pain, especially in the right lower quadrant, with a low-grade fever (temperature $37.7^{\circ} \mathrm{C}$ ). His $\mathrm{CBC}$ showed an ANC $0.00 \times 10^{9} / \mathrm{L}$, hemoglobin of $8.6 \mathrm{~g} / \mathrm{L}$, and platelet count of $43 \times 10^{9} / \mathrm{L}$. An abdominal CT showed a long, tubular appendix with wall thickening. Because of his pancytopenic state, antibiotic therapy with prepenem was given first. Over the course of antibiotic therapy, the intensity of the patient's pain decreased and his general condition improved. After 7 days, we performed a follow-up abdominal CT scan, which showed an interval decrease of the inflammatory process of acute appendicitis with periappendiceal inflammatory change. Chemotherapy was continued as scheduled. While waiting for a bone marrow transplant, the 

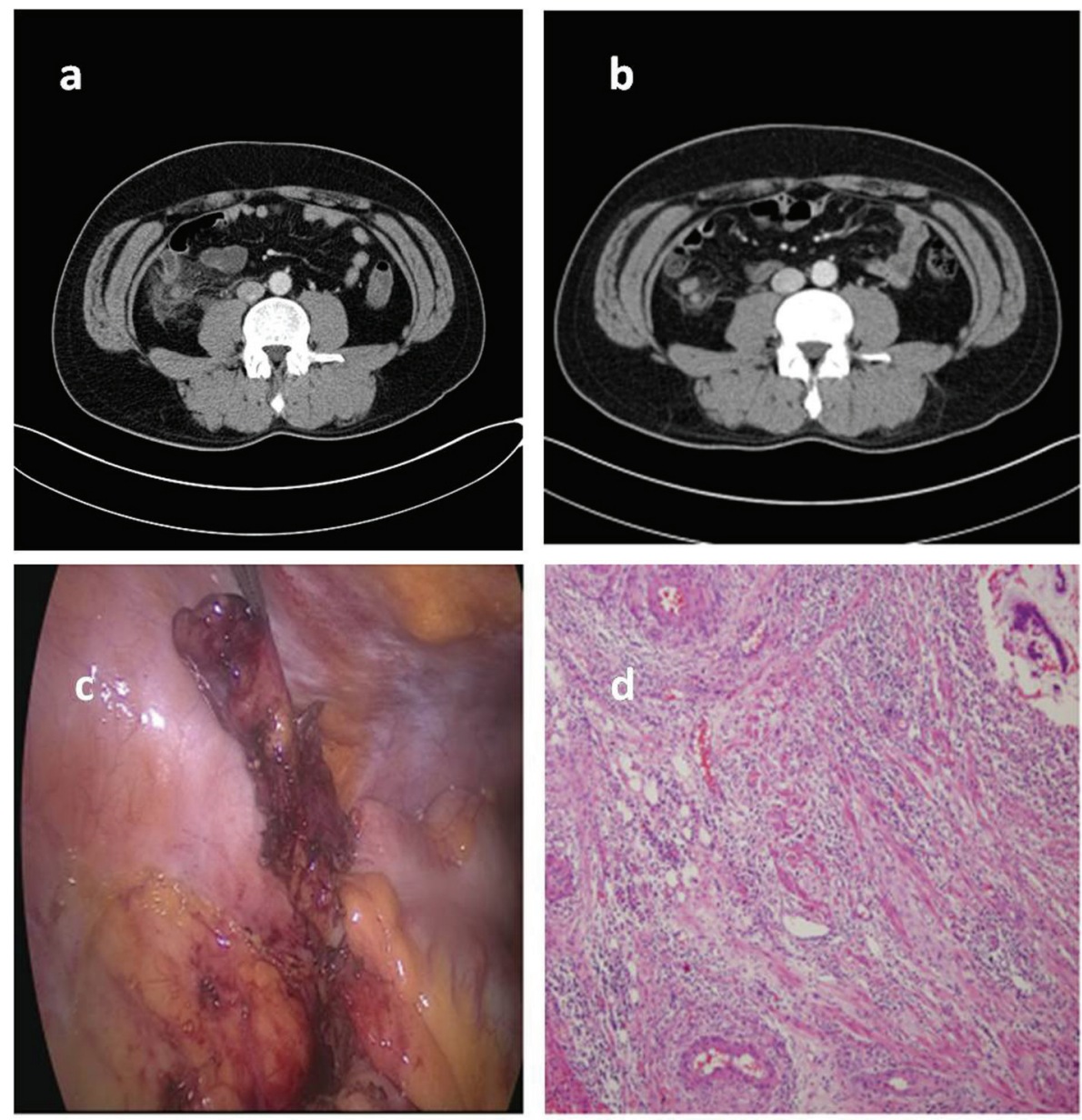

Figure 2. Radiologic, laparoscopic and histologic features of acute appendicitis in an neutropenic leukemic patient. Abdominal CT showed wall thickening and edema of the appendix and periappendiceal inflammatory change (a). Abdominal CT showed an interval decrease of inflammatory process of acute appendicitis with periappendiceal inflammatory change (b). A laparoscopic appendectomy was performed (c), and histologic examination of the appendix revealed infiltration of fibrin and inflammatory cells, without leukemic infiltration (hematoxylin-eosin, low magnification $\times 100)(d)$.

patient underwent a laparoscopic appendectomy (Fig. 2). No perioperative complications occurred. The patient was able to take oral fluids on POD 1. The patient was discharged on POD 2 and continued his scheduled chemotherapy with subsequent consolidations.

\section{Discussion}

Abdominal pain in neutropenic patients requires rapid diagnosis. The clinical presentation of abdominal pain in neutropenic patients can be indicative of a wide variety of diagnoses and may require surgery. Badgwell et al reported on 60 patients with neutropenia and symptoms of abdominal pain. The median ANC of these patients was $0.17 \times 10^{9} / \mathrm{L}$, and their median platelet count was $29.5 \times 10^{9} / \mathrm{L}$. The causes of abdominal pain were necrotizing enterocolitis $(n=17 ; 28 \%)$, small bowel obstruction $(\mathrm{n}=7 ; 12 \%)$, diverticulitis $(\mathrm{n}=3$;
$5 \%)$, appendicitis $(\mathrm{n}=3 ; 5 \%)$, cholecystitis $(\mathrm{n}=2 ; 3 \%)$, colonic pseudo-obstruction $(\mathrm{n}=2 ; 3 \%)$, splenic rupture $(\mathrm{n}=1$; $2 \%$ ), and no definite cause ( $\mathrm{n}=21 ; 35 \%)$. These diagnoses can be difficult to distinguish using imaging technology [6]. In our case, patients underwent abdominal CT to confirm the diagnosis. Abdominal surgery may result in significant morbidity or mortality in neutropenic patients [3-5]. Some authors have concluded that, if possible, the disease should be treated with intensive medical therapy rather than surgery [3].

However, surgical treatment is sometimes unavoidable. In patients with neutropenia, it is important to determine when surgery is safest. However, it is difficult to compare surgical management with non-surgical management, and this to determine whether to delay or avoid surgery in the neutropenic period. There are various opinions on the treatment of acute appendicitis in neutropenic patients. An initial nonoperative antibiotic treatment has been reported to 
often be successful in appendicitis [1]. In contrast, medical therapy as the sole treatment has been associated with longer hospital stays and recurrent appendicitis or complications [7]. Acute appendicitis usually requires surgical intervention [7-9]. In this paper, we report two cases of appendectomy in adult neutropenic patients with hematologic disease. Initially, the patients' conditions were not suitable for surgical intervention, so antibiotics and donor granulocyte infusion (DGI) were initiated. With thoroughly prepared supportive care, delayed surgery was successfully completed with no acute or late complications. We suggest that, in patients in a neutropenic state, delayed elective operation is superior to urgent operation, except in the case of life-threatening complications such as peritonitis. When possible, a laparoscopic approach should be considered because of the significantly lower rate of wound infection and hemorrhagic post-surgical complications $[9,10]$.

\section{Acknowledgement}

This research was supported by Seoul St. Mary's Clinical Medicine Research Program year of 2011 through the Catholic University of Korea.

\section{Conflict of Interest}

The authors declare that they have no conflict of interest.

\section{References}

1. Wiegering VA, Kellenberger CJ, Bodmer N, Bergstraess- er E, Niggli F, Grotzer M, Nadal D, et al. Conservative management of acute appendicitis in children with hematologic malignancies during chemotherapy-induced neutropenia. J Pediatr Hematol Oncol. 2008;30(6):464467.

2. Schaller RT, Jr., Schaller JF. The acute abdomen in the immunologically compromised child. J Pediatr Surg. 1983;18(6):937-944.

3. Scott-Conner CE, Fabrega AJ. Gastrointestinal problems in the immunocompromised host. A review for surgeons. Surg Endosc. 1996;10(10):959-964.

4. Skibber JM, Matter GJ, Pizzo PA, Lotze MT. Right lower quadrant pain in young patients with leukemia. A surgical perspective. Ann Surg. 1987;206(6):711-716.

5. Wade DS, Douglass H, Jr., Nava HR, Piedmonte M. Abdominal pain in neutropenic patients. Arch Surg. 1990;125(9):1119-1127.

6. Hobson MJ, Carney DE, Molik KA, Vik T, Scherer LR, 3rd, Rouse TM, West KW, et al. Appendicitis in childhood hematologic malignancies: analysis and comparison with typhilitis. J Pediatr Surg. 2005;40(1):214-219; discussion 219-220.

7. Ozyurek E, Arda S, Ozkiraz S, Alioglu B, Arikan U, Ozbek N. Febrile neutropenia as the presenting sign of appendicitis in an adolescent with acute myelogenous leukemia. Pediatr Hematol Oncol. 2006;23(3):269-273.

8. WK Kang, SD Kim, HJ Kim, HM Cho, SC Park, HM Jeon, et al. Right low quadrant pain in patient with acute myeloid leukemia. J Korean Surg Soc 2004;66:420-423.

9. Ustun C. Laparoscopic appendectomy in a patient with acute myelogenous leukemia with neutropenia. J Laparoendosc Adv Surg Tech A. 2007;17(2):213-215.

10. Sweeney KJ, Keane FB. Moving from open to laparoscopic appendicectomy. Br J Surg. 2003;90(3):257-258. 